\title{
COVID-19 detection using cough sound analysis and deep learning algorithms
}

\author{
Sunil Rao ${ }^{\mathrm{a}, *}$, Vivek Narayanaswamy ${ }^{\mathrm{a}}$, Michael Esposito ${ }^{\mathrm{a}}$, Jayaraman J. Thiagarajan ${ }^{\mathrm{b}}$ and \\ Andreas Spanias ${ }^{\mathrm{a}}$ \\ ${ }^{a}$ School of ECEE, SenSIP Center, Arizona State University, Tempe, AZ, USA \\ ${ }^{\mathrm{b}}$ Lawrence Livermore Nat. Labs, Livermore, CA, USA
}

\begin{abstract}
Reliable and rapid non-invasive testing has become essential for COVID-19 diagnosis and tracking statistics. Recent studies motivate the use of modern machine learning (ML) and deep learning (DL) tools that utilize features of coughing sounds for COVID-19 diagnosis. In this paper, we describe system designs that we developed for COVID-19 cough detection with the long-term objective of embedding them in a testing device. More specifically, we use log-mel spectrogram features extracted from the coughing audio signal and design a series of customized deep learning algorithms to develop fast and automated diagnosis tools for COVID-19 detection. We first explore the use of a deep neural network with fully connected layers. Additionally, we investigate prospects of efficient implementation by examining the impact on the detection performance by pruning the fully connected neural network based on the Lottery Ticket Hypothesis (LTH) optimization process. In general, pruned neural networks have been shown to provide similar performance gains to that of unpruned networks with reduced computational complexity in a variety of signal processing applications. Finally, we investigate the use of convolutional neural network architectures and in particular the VGG-13 architecture which we tune specifically for this application. Our results show that a unique ensembling of the VGG-13 architecture trained using a combination of binary cross entropy and focal losses with data augmentation significantly outperforms the fully connected networks and other recently proposed baselines on the DiCOVA 2021 COVID-19 cough audio dataset. Our customized VGG-13 model achieves an average validation AUROC of $82.23 \%$ and a test AUROC of $78.3 \%$ at a sensitivity of $80.49 \%$.
\end{abstract}

Keywords: COVID-19 detection, machine learning, deep learning, respiratory diagnosis, healthcare, artificial intelligence

\section{Introduction}

With the outbreak of the COVID-19 pandemic caused by the coronavirus SARS-CoV-2, the severity of the infection and the associated fatality rates around the world are increasing at an alarming rate [1]. Although reverse transcriptase-polymerase chain reaction (RTPCR) testing is generally adopted, coughing sounds have been found to reveal useful signatures pertaining to COVID-19 which can be used to facilitate rapid, noninvasive, and reliable screening methodologies [2]. Consequently, spectral and waveform signatures from

${ }^{*}$ Corresponding author: Sunil Rao, School of ECEE, SenSIP Center, Arizona State University, 650 E Tyler Mall, Tempe, AZ 85281, USA. E-mail: rsunilsr@asu.edu. coughing patterns associated with the disease are being considered as biomarkers for prognosis. In this context, there are several initiatives towards enabling open research on COVID-19 detection from coughing sounds. For example, the authors of [3] curated the Coswara dataset consisting of cough samples collected from subjects who either tested positive or negative for COVID19. Similarly, the COUGHVID dataset described in [4] is comprised of crowd-sourced cough samples collected from COVID-19 positive and negative patients across a wide range of demographic backgrounds. These efforts naturally pave way to the development of diagnosis tools powered by machine learning (ML) and deep learning (DL) for detecting COVID-19 from cough sounds. Although ML has been extensively applied for a variety of speech and audio processing applica- 


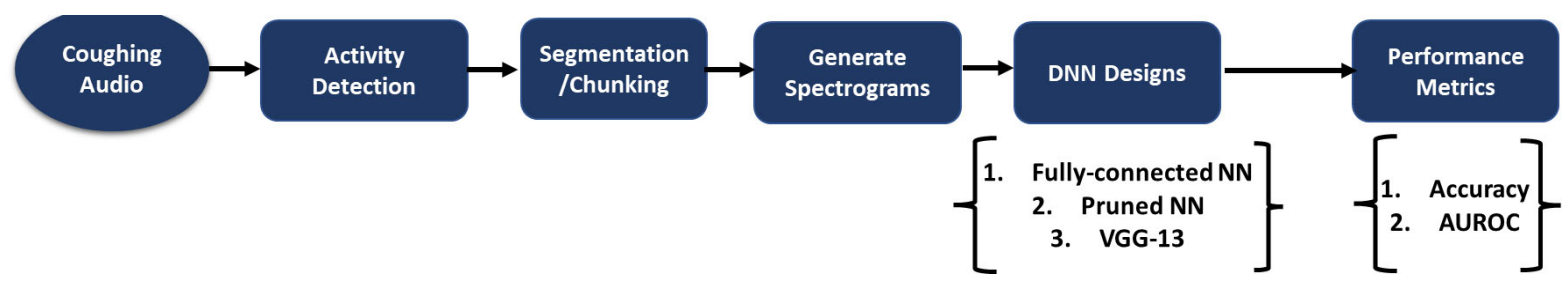

Fig. 1. A block diagram depicting an overview of the system used for COVID-19 detection using cough samples. The system uses a unique combination of cross entropy and focal loss functions which yield improved performance with COVID-19 audio.

tions [5-12], there have been new studies [13-25] that specifically address audio analysis for COVID-19. Applications of these studies range from implementation of COVID-19 audio detection software for individual use to widespread pandemic monitoring across communities and cities. The authors of [18] gathered audio samples of coughing and breathing through the "COVID-19 Sounds" app and demonstrated the feasibility of using ML based tools for COVID-19 diagnosis. In [23], it was shown that biomarkers originally developed for Alzheimer's disease characterization could be repurposed for designing COVID-19 detectors. Similarly, the authors of [24] identified a novel set of biomarkers from coughs of COVID-19 patients and utilized deep neural networks to design predictive models. The majority of these studies utilize deep neural networks (DNNs) for classification in lieu of conventional ML algorithms owing to the need for accurately characterizing the complex decision boundaries between spectral signatures of COVID-19 positive and negative subjects.

Given the complex nature of coughing audio spectral signatures, there is a need to systematically develop architectural designs [29] that can effectively leverage the audio features for accurate detection (Fig. 1). To this end, in this paper, we describe DL system designs that we developed to classify cough samples from the DiCOVA 2021 dataset [3] into two categories, namely: healthy (COVID-19 negative) and unhealthy (COVID19 positive). We begin by investigating the use of a DNN with fully connected layers for cough detection. With an intent to import such DNN models for COVID19 cough detection on mobile and edge-devices, we prune the neural network based on the Lottery Ticket Hypothesis (LTH) [28] optimization process. Pruned neural networks have been explored for various applications in energy, signal, and image processing [26-28] and have shown to provide similar performance gains with unpruned networks yet yield computationally efficient implementations. Finally, we investigate the impact of convolutional neural network (CNNs) architectures, in particular VGG-13 [30] for detection. Interestingly, based on extensive empirical analyses, we find that a unique ensembling of the VGG-13 architecture trained using a combination of binary cross entropy and focal losses [39] with minority class oversampling (COVID-19 positive) and data augmentation (addition of samples from an auxiliary dataset) significantly outperforms the fully connected networks and other recently proposed baselines on the DiCOVA 2021 COVID-19 cough audio dataset. Our proposed convolutional model achieves an average validation AUROC of $82.23 \%$ and a test AUROC of $78.3 \%$ at a sensitivity of $80.49 \%$ evidencing the efficacy of the proposed approach for COVID-19 cough detection.

The rest of the paper is organized as follows: We first describe the related work in this area of research in Section 3 followed by pre-processing and feature extraction strategies adopted in our work in Section 4. We then describe in detail the design methodologies adopted beginning from a general fully connected DNN (Section 5), pruned networks based on LTH (Section 6) followed by the proposed, customized VGG-13 architecture for cough detection (Section 7). We also describe our approaches to handle imbalanced data by a) using up-sampling, b) modifying the loss function and c) employing ensemble strategies. We discuss our findings and inferences in Section 8 , and we close with concluding remarks in Section 9.

\section{Related work}

Diagnosis of COVID-19 by cough sound analysis is plausible due to the fact that the coughing patterns are reflective of the physical alterations to the lungs and diaphragm $[32,33]$ which are predominantly targeted by the virus. As a result, there have been several efforts [2-4,18,22,34-36] for detecting signatures of COVID-19 from cough samples using ML tools. Brown et al. [18] used ML based algorithms to distinguish between healthy and COVID-19 cough sounds using crowdsourced data. The authors gathered data using their own "COVID-19 Sounds" web and Android app where 141 COVID-19 samples and 350 healthy 
samples were selected for training. Classical audio features including root mean square energy (RMSE), mel-frequency cepstral coefficients (MFCCs) and spectral centroids were used for training logistic regression/support vector machines (SVMs) classifier models. The authors report an average AUROC metric of $80 \%$ for the different tasks reported in their study. Subsequent to this effort, the Coswara project [3] compiled a crowdsourced dataset containing a variety of sounds including sustained phonations, counting, coughs and breathing patterns. Also, multiple types of coughs and breathing sounds were collected from the users. The Coswara project was one of the first publicly available COVID-19 audio datasets and remains unique in its wide variety of sounds collected. Utilizing classical features such as MFCCs [37,38], spectral centroid and mean square energy to train a random forest classifier for the sound classification task, the authors report a test accuracy of $66 \%$. More recently, Imran et al. [2] developed tools that utilize CNNs trained with mel spectrograms for cough detection followed by model ensembling to determine whether or not the sample belonged to a COVID-19 patient. In this study, the authors gathered cough samples from patients diagnosed with COVID-19, bronchitis, and pertussis. 48 COVID-19, 102 bronchitis, 131 pertussis, and 76 healthy samples were used in order to train their algorithm. The algorithm consisted of three separate classifiers, namely, a deep learning-based multi class classifier, classical ML based multi class classifier, and deep learningbased binary class classifier. The authors report accuracies of $93.56 \%, 94.06 \%$, and $88.89 \%$ for these classifiers, respectively. They also developed an app, "AI4COVID-19", in order to allow users to interface with their AI-based COVID-19 detection algorithm. A recent study [27] by MIT's Open Voice Team has further validated the practicality of detecting COVID-19 using cough samples powered by deep neural networks and have reported significantly higher sensitivity and specificity metrics. Their model was trained and verified using 2,600 COVID-19 positive audio samples and 2,600 COVID-19 negative audio samples. The authors extract MFCC features which are then passed through a CNN architecture built upon the designs in $[39,40]$. At a sensitivity of $98.5 \%$ and a specificity of $94.2 \%$, the authors achieved and AUROC metric of 97\%. Similarly, the authors from the Virufy consortium [41] have employed an ensemble of three neural networks trained on mel-spectrograms of cough audio to perform the detection. The algorithm is reported to predict COVID19 infection with an AUROC of $77.1 \%$ which further motivates the use of deep neural network models for carefully discriminating between the types of cough sounds. In our previous work [22], we use an ensemble of four deep models namely CNNs, Recurrent Neural Networks (RNNs), Gated CNNs (GCNNs), Gated Convolutional Recurrent (GCRNNs) for COVID-19 cough detection on the DiCOVA 2021 dataset. However, the average validation accuracy obtained using the stacked approach was only $65 \%$. In this paper however, we find that in addition to model ensembling or fusion strategies, the careful choice of loss functions and meaningful data augmentations was critical in improving the overall generalization performance (improved accuracies and AUROC scores on the validation folds and the blind test dataset) of the VGG-13 convolutional architecture under limited data scenarios.

\section{Pre-processing and feature extraction}

\subsection{Description of the DiCOVA dataset}

The DiCOVA Challenge [46] was designed to find scientific and engineering insights on COVID-19 by analyzing acoustic cues gathered from COVID-19 positive and non-COVID-19 individuals. The goal is to use cough sound recordings from COVID-19 and nonCOVID-19 individuals for the task of COVID-19 detection. The train dataset contains a total of $\sim 1.36 \mathrm{hrs}$ of cough audio recordings from 75 COVID-19+ve subjects and 965 non-COVID-19 subjects. Out of these samples, there were a total of five splits for training and validation. The challenge also required the participants to evaluate the models on a blind test dataset.

\subsection{Silence removal and downsampling}

We first perform silence removal on the given cough audio files. If the duration of silence was greater than $500 \mathrm{~ms}$, we split the audio wave file at that corresponding instance. Similarly, if the amplitude of the wave files was less than $-48 \mathrm{~dB}$, we remove the corresponding part of the wave file. After silence removal, we perform down sampling of the audio files to $32 \mathrm{kHz}$.

\subsection{Feature extraction}

We transform the pre-processed wave files into the spectral logmel scale. Log-mel features are a popular choice for classification using neural networks as they benefit from the additional information such as rich 


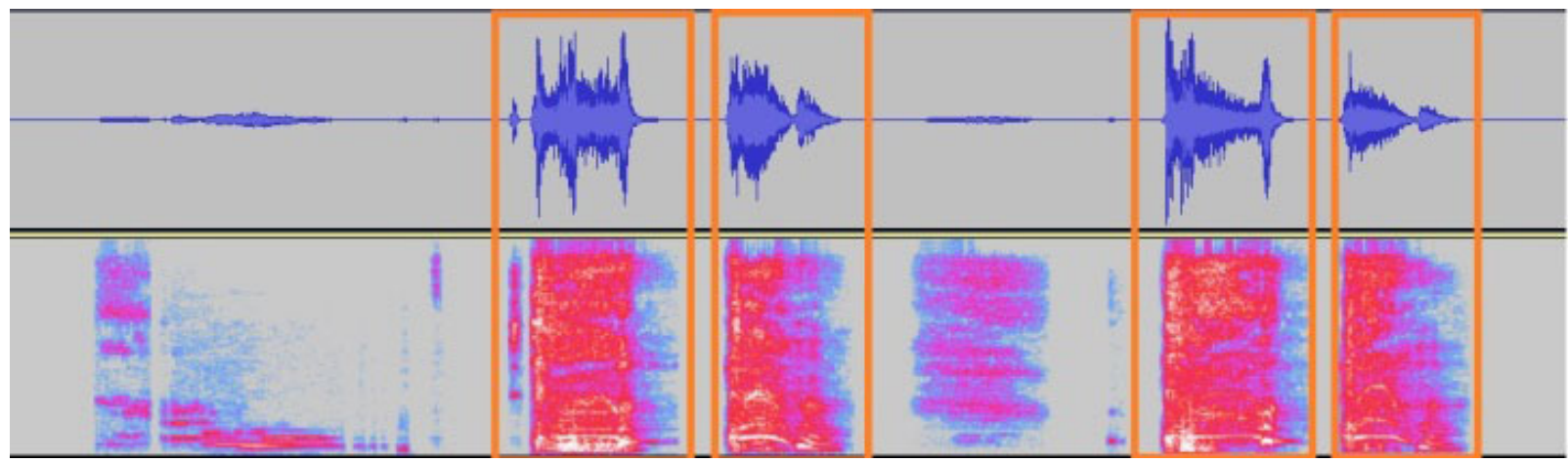

Fig. 2. A sample audio file split into $1 \mathrm{~s}$ segments with silence removed. The corresponding spectrograms are passed to the neural network.

Table 1

Parameters used to obtain the log-mel spectrogram

\begin{tabular}{ll}
\hline Parameter & Configuration \\
\hline Sample rate & $32000 \mathrm{~Hz}$ \\
Window size & 512 \\
Hop size & 256 \\
Mel bands & 64 \\
\hline
\end{tabular}

temporal and spectral structure of the original signal. These wave files are chunked into excerpts of duration $\sim 1 \mathrm{~s}$. If the excerpt is less than $1 \mathrm{~s}$, the log-mel spectrogram is zero padded. We choose a window size of 512 and hop size of 256 to correspond to the $1 \mathrm{~s}$ spectrogram. Therefore, each spectrogram has a size of $128 \times 64$. Table 1 shows the parameters used to obtain the log-mel spectrogram. Figure 2 shows an audio file being split into multiple $1 \mathrm{~s}$ chunks, and the extraction of its corresponding spectrogram. The spectrograms of size $128 \times 64$ are passed as input to the neural network classifier.

\subsection{Experimentation with additional features}

Prior to choosing the log-mel spectrogram, we investigated the use of classical features [42] such as the frame level MFCCs with the delta and delta-delta coefficients, RMS energy, Spectral Centroid (SC), Spectral Roll-off (SR), Spectral-Bandwidth (SB) and the Zero-Crossing Rate (ZCR) in classifying the cough sounds as either belonging to a COVID-19 positive or a healthy patient. In particular, we concatenated these statistical features and utilized an XGBoost [58] model to perform the classification. However, we found that despite using sophisticated model selection strategies, namely 5-fold cross validation and hyperparameter tuning, the generalization even to the validation data was not sufficient. We found that the model predicted almost all of the COVID-19 positive samples as belonging to the healthy class. We also observed that the other classification metrics (AUROC, Specificity) was found to be $55 \%$ and $40 \%$ respectively which naturally motivated the choice for a more powerful feature extractor. We observed similar performances with the use of a feed-forward neural network when trained with these features. Studies in [31] show that the log-mel spectrograms outperform traditional representations such as MFCCs for deep neural network architectures. In the following section we describe the use of a fully connected neural network for COVID-19 cough detection where the $\mathrm{NN}$ is trained using the log-mel spectrograms.

\section{Detectors based on fully connected neural networks}

In this paper, we consider the COVID-19 cough detection as a supervised learning task. For the fully connected neural network, consider a dataset $\{X, y\}$ where $X \in R^{m \times n}$ where $m$ is the total number of samples and $n$ is the input feature dimension; $y \in\{0,1\}$ is the target label of whether the sample belongs to the COVID-19 negative (0) or the positive (1) class. In order to feed the cough audio spectrograms into a fully connected network, we vectorize (flatten) and stack the spectrograms to obtain a dataset $X \in R^{6385 \times 8192}$. Using the input spectrogram as features, we feed them as inputs to a fully connected neural network, popularly referred to as the multi-layer perceptron (MLP). We use a 5 layered neural network of hidden layer dimensions 50, 100, 500, 1000 respectively. Information flows through the neural networks in two ways: (i) In forward propagation the MLP model predicts the output for the given data and (ii) In backpropagation the model adjusts its parameters considering the error in the prediction. The activation function used in each neuron allows the 


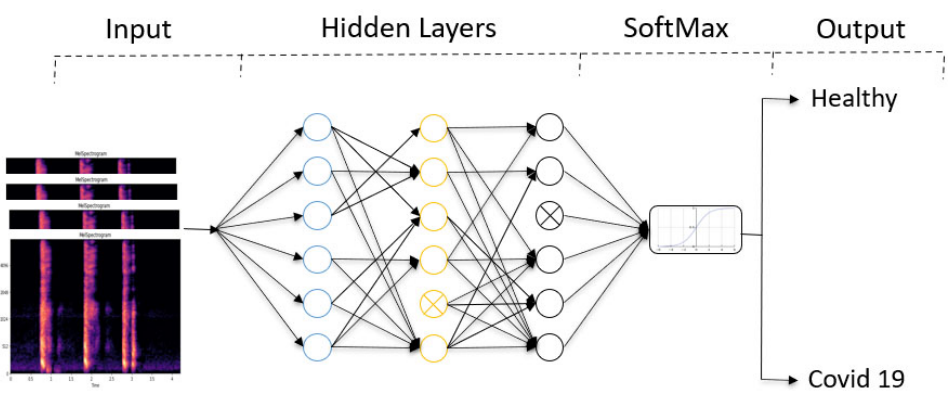

Fig. 3. A figure depicting a pruned neural network using the Lottery Ticket Hypothesis.

MLP to learn a complex function mapping. Input to the model is the feature vector $x \in X$, the output of the first and consecutive hidden layer is given by

$$
\begin{aligned}
& h_{1}=\sigma\left(W_{1} \cdot x+b_{1}\right) \\
& h_{i}=\sigma\left(W_{i} \cdot h_{i-1}+b_{i}\right)
\end{aligned}
$$

where $i$ is the layer index and $\sigma$ is the activation function. The final output of the MLP is obtained as:

$$
\hat{y}=\phi_{\text {softmax }}\left(h_{\text {out }}\right)
$$

Weights of each neuron are trained/updated using a scaled gradient backpropagation algorithm. Each layer is assigned a tanh (hyperbolic tangent) activation function. From our experiments, we find that the tanh activation function provided the best accuracy. The output layer uses the SoftMax activation function to categorize the type of cough in the given dataset. We utilize the given train and validation splits of the DiCOVA dataset to train and evaluate the model. Using a fully connected neural network, we observed an average accuracy of $\sim$ $65 \%$ on the given validation splits. Consequently, we explored pruning the neural network to develop sparse or compressed neural networks to understand whether similar performance gains can be expected. The use of sparse neural networks can be potentially used in development of COVID-19 detection software on hardware and cloud applications. We discuss the method in the next section.

\section{Pruned neural networks using lottery ticket hypothesis}

Pruned NNs on embedded hardware significantly provides computational efficiencies and reduce memory requirements with only with slight reduction in accuracy in comparison with the unpruned networks. A popular strategy to prune $\mathrm{NN}$ is based upon the Lottery Ticket Hypothesis [28]. It is based upon the idea that a randomly initialized, dense network contains a subnetwork initialized such that when trained in isolation it can match the test accuracy of the original network after training for at most the same number of iterations. Consider a fully connected $\mathrm{NN}$ with $\mathrm{N}$ neurons in each layer initialized by weight matrices $W^{0}=\left[W_{i}^{0}\right]_{i=1}^{L}$. After training this network for $t$ epochs, the resulting weights of the network are $W^{t}$. Next, compute a mask $M$ by pruning $p \%$ of the of weights closer to zero by taking the absolute value. Reinitialize the network with $W^{0}$ masked by $M$. The network training and network pruning process is iterated until $2.5 \mathrm{x}$ compression is achieved, after which the networks performance degrades due to underfitting of the data.

- Randomly initialize a neural network $f(x ; m \bigodot \theta)$ where $\theta=\theta_{0}$ and $m=1^{|\theta|}$ is a mask.

- Train the network for $j$ iterations, reaching parameters $m \bigodot \theta_{j}$.

- Prune $s \%$ of the parameters, creating an updated $m^{\prime}$ where $P_{m^{\prime}}=\left(P_{m}-s\right) \%$.

- Reset the weights of the remaining portion of the network to their values in $\theta_{0}$. That is, let $\theta=\theta_{0}$.

- Let $m=m^{\prime}$ and repeat steps 2 to 4 until a sufficiently pruned network has been obtained.

For the network pruning experiments, we consider $\mathrm{NNs}$ with four hidden layers each with $\mathrm{N}=\{50,100$, 500, 1000\} neurons (Fig. 3). All NNs were trained for 150 epochs and at every pruning iteration $10 \%$ of the remaining weights were pruned. We find that pruning a network for this setup degrades the performance of the classification task as shown in Fig. 4. While utilizing fully connected neural networks, the inputs are essentially vectors of the spectrogram samples. The architecture of these NNs is such that they do not consider local spatio-temporal and periodic patterns highly prevalent in audio spectrogram data. Therefore, in order to exploit these patterns, we explore the use of deep convolutional neural network architectures which by design can identify markers in audio critical to distinguish between 


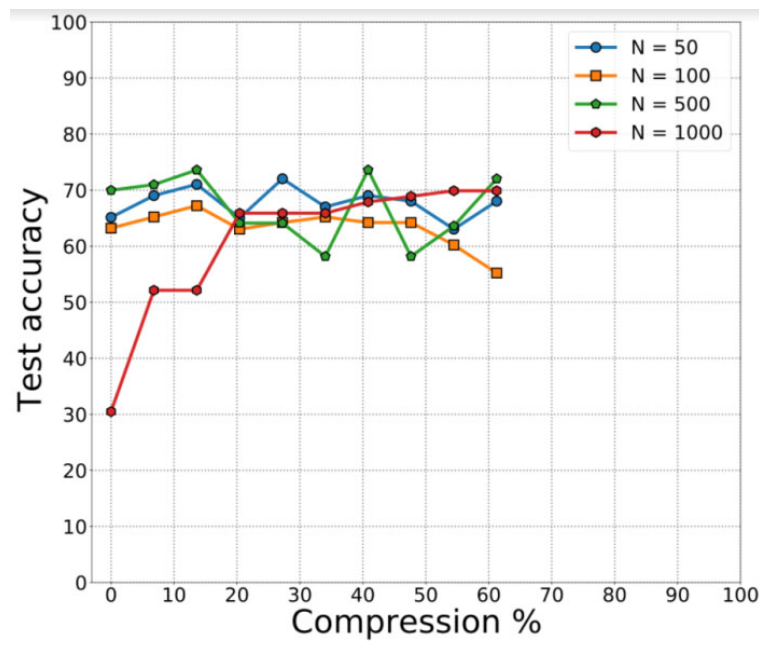

Fig. 4. Results depicting pruned neural networks for covid audio classification. The pruning across the dataset degrades the performance of the classification task.

healthy (COVID-19-ve) and unhealthy (COVID-19+ve) cough sounds.

\section{Customizing the VGG-13 architecture}

We utilize a VGG-13 [30] architecture to train and evaluate performance over the different training and validation splits in the DiCOVA 21 challenge dataset. The VGG architecture has been successfully used for image recognition and audio classification audio classification applications $[59,60]$. VGG-13 consists of multiple convolutional blocks followed by a fully connected network to make the final predictions. Each convolutional block consists of two convolutional layers followed by a max pooling layer that halves each of the spatial dimensions. Every convolution layer is followed by a ReLU activation function and batch normalization [43] which is a form of regularization to tackle internal covariate shifts. After the convolutional blocks, the resulting feature maps are averaged pooled and fed as inputs to a feed forward neural network. Finally, a SoftMax layer is used to generate the predictions. The size of the spectrogram that is used as input to the VGG network is $128 \times 64$. Spectrograms were generated from each excerpt after suitable pre-processing. An overview of the overall system is shown in Fig. 5.

Due to the imbalance in the DiCOVA dataset across the healthy and COVID-19 positive classes, through extensive empirical studies, we find model ensembling and data augmentation strategies to improve model generalization. The term ensembling here implies the use of the best two models for each loss function. We then obtain the output by averaging the probability scores from each model. In this work, we ensemble two models for our predictions. To train the first model, we use the DiCOVA dataset and augment with the COVID-19 positive samples from the COUGHVID dataset [4] and up-sample the training data to maintain data balance. We use a cross entropy loss to train this model. To train the second model, we perform data augmentation similar to that of the first model except up-sampling and use focal loss [44]. These methods are described in more detail below.

\subsection{Data augmentation}

Since the DiCOVA 2021 dataset has only 50 COVID19 positive samples per validation fold, we augment it with the COUGHVID dataset to train the network. We use 400 COVID-19 positive samples from the COUGHVID dataset for network training. To avoid overfitting during training, we utilize an additional data augmentation strategy. We use the audio mix-up [45] method to achieve the same. Mix-up randomly mixes a pair of inputs and their corresponding outputs. Consider a pair of inputs $x_{1}$ and $x_{2}$ with $y_{1}$ and $y_{2}$ being their corresponding labels. Mix-up uses a parameter $\lambda \in(0$, 1) to create a convex combination as shown below:

$$
\begin{aligned}
& x=\lambda x_{1}+(1-\lambda) x_{2} \\
& y=\lambda y_{1}+(1-\lambda) y_{2}
\end{aligned}
$$

Mixup operates on the fly by randomly mixing a pair of inputs and their associated target values. The output of Eqs (4) and (5) were used to train the network rather than the original inputs. The parameter $\lambda$ was chosen to be a random variable from the uniform distribution $U \sim(0,1)$.

\subsection{Loss functions}

We train two models based on two different loss function choices. One model is trained using the crossentropy loss while the second model is trained using the focal loss. We ensemble these two models to generate the probabilities on the validation and test datasets.

\subsubsection{Cross entropy loss}

To address data imbalance, we augment the COUGHVID dataset and up sample the training data for each training fold. We balance the number of samples in both classes in the training data through up sampling. The up-sampled training data is used to train the VGG- 


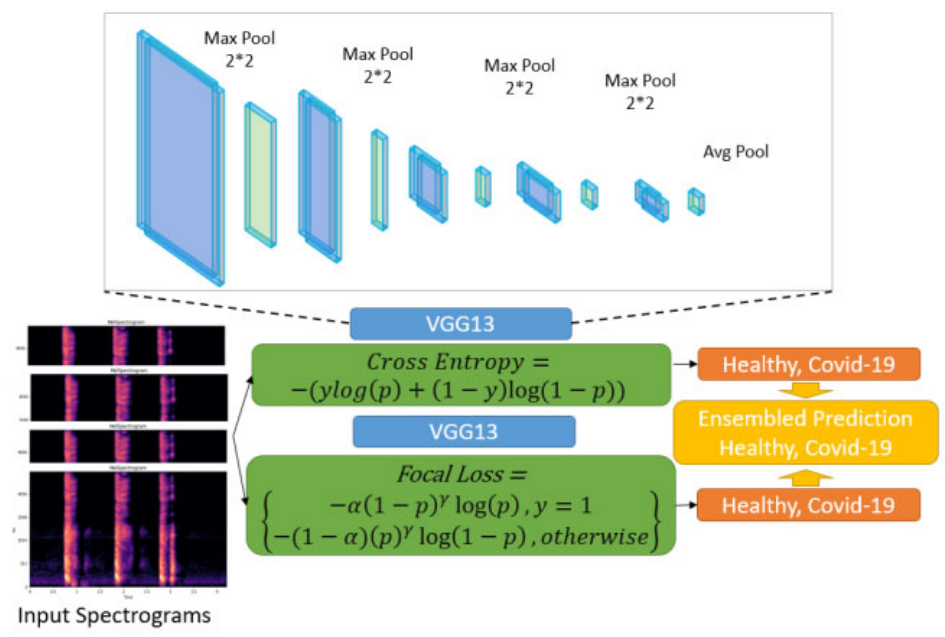

Fig. 5. The overview of the model used for COVID-19 detection using VGG-13. Each VGG-13 model is trained with combined cross entropy loss and focal loss. The final results are obtained after integrating the two models.

13 model using the cross-entropy loss. The binary cross entropy loss is given as:

$$
C E=-y \log (p)-(1-y) \log (1-p)
$$

where $y \in\{0,1\}$ corresponds to the label of the two classes and $p$ is the probability of the outputs.

\subsubsection{Focal loss}

Focal loss is used to address the class imbalance problem [39]. Two modulation hyper-parameters $\alpha$ and $\gamma$ are added to the cross-entropy loss in Eq. (6) to make it efficient to the imbalanced class problem. We then include 400 all the COVID-19 positive samples from COUGHVID in each training fold containing $50 \mathrm{Di}-$ COVA COVID-19 positive samples. We then use the focal loss with modulation parameters $\alpha=025$ and $\gamma=2$ to train the network.

$$
\begin{aligned}
F L=\{ & -\alpha \log (p)(1-p)^{\gamma} y=1 \\
& \left.-(1-\alpha) \log (1-p) p^{\gamma} \text { otherwise }\right\}
\end{aligned}
$$

The parameters alpha and beta were finalized through extensive experimentation using grid search. Gridsearch was used to determine the optimal hyperparameters of a model which results in the most 'accurate' predictions. Focal loss applies a modulating term to the cross-entropy loss in order to focus learning on hard negative examples. It is a dynamically scaled cross entropy loss, where the scaling factor decays to zero as confidence in the correct class increases. Intuitively, this scaling factor can automatically down-weight the contribution of easy examples during training and rapidly focus the model on hard examples. We introduced the focal loss in training to address the issue of data imbalance in the given dataset.

\subsubsection{Ensembling}

We use the two models trained with the loss functions above. We use the predictions from the individual models on the five validation folds and on the test dataset. We finally obtain the prediction probabilities for all validation/test samples by averaging the predictions from the two models. We describe the results in detail below in Section 8.

\section{Results}

\subsection{Benchmarking different design strategies}

For the fully connected neural networks, we obtain an average validation accuracy of $65 \%$ on the 5 folds provided. In case of the pruned neural networks for cough detection, we observe a similar classification accuracy of $64.3 \%$. We find that smaller networks achieve greater compression of about $60 \%$ for a drop in accuracy by $7 \%$. We then explored more sophisticated convolutional neural network architectures such as the VGG-13. We first discuss the results obtained from VGG-13 models before and after ensembling. For the first model, which was trained using the DiCOVA dataset augmented with COUGHVID data for each of the five folds with crossentropy loss, we obtain an average AUROC of $84.02 \%$ on the five folds. The test AUROC was found to be $73.48 \%$. For the second model, which followed a similar augmentation strategy as the first model except up sampling and focal loss, we find an average validation AUROC of $72.01 \%$ on the five folds. The test AUROC was found to be $73.44 \%$. We summarize these results in 
Table 2

AUROC for each individual fold of the validation dataset with combined cross entropy loss and focal loss with highest specificity for each fold

\begin{tabular}{ccc}
\hline Validation fold & $\begin{array}{c}\text { AUROC under } \\
\text { cross entropy }(\%)\end{array}$ & $\begin{array}{c}\text { AUROC under } \\
\text { focal loss }(\%)\end{array}$ \\
\hline 1 & 80.71 & 70.27 \\
2 & 89.88 & 68.76 \\
3 & 87.12 & 77.78 \\
4 & 73.59 & 70.52 \\
5 & 94.92 & 72.93 \\
\hline
\end{tabular}

Table 3

A table comparing the validation AUROCs of the methods used for COVID-19 audio detection in the DiCOVA 2021 challenge

\begin{tabular}{lc}
\hline \multicolumn{1}{c}{ Method } & AUROC \\
\hline Light gradient boosting [47] & 67.28 \\
SVM and LSTM [48] & 94.31 \\
Random forest and SVM [49] & 73.29 \\
Logistic regression and random forest [50] & 73.41 \\
Autoregressive predictive coding [51] & 76.45 \\
Autoencoder [52] & 47.28 \\
SVM [53] & 71.7 \\
Resnet18 [54] & 61.9 \\
VGG-13 (our method) & 82.23 \\
\hline
\end{tabular}

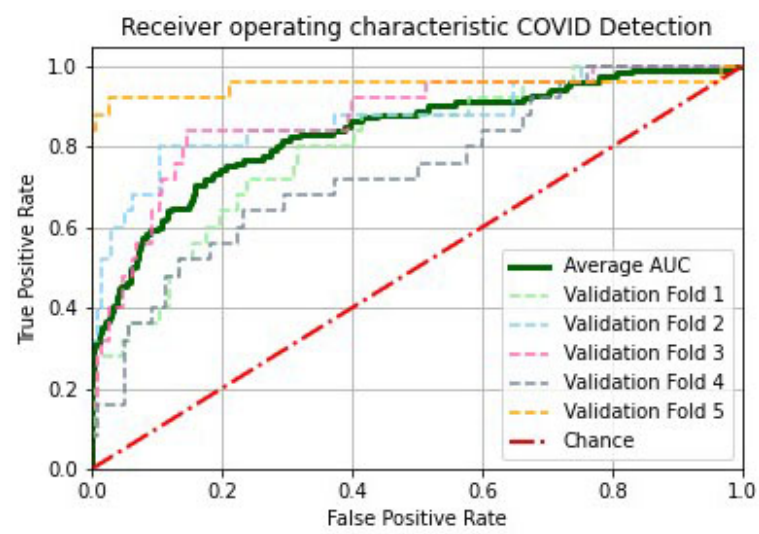

Fig. 6. Average validation AUROC curve on the five folds. We observed that our proposed approach yielded an AUROC score of $82.28 \%$.

Table 2. Since the individual models reported low AUROC scores on the test dataset, we predict the probability scores on the best of the two models. i.e., to obtain an improved AUROC score, we select and ensemble the two best models for each loss function and from each fold and predict on the corresponding validation fold to obtain the ensembled AUROC. Following such an ensembling strategy, we notice that the average validation AUROC increases to $82.23 \%$ (as observed from Fig. 6) on the validation folds while the AUROC on the blind test dataset was found to be $78.3 \%$.

Figure 7 depicts the confusion matrix obtained at a

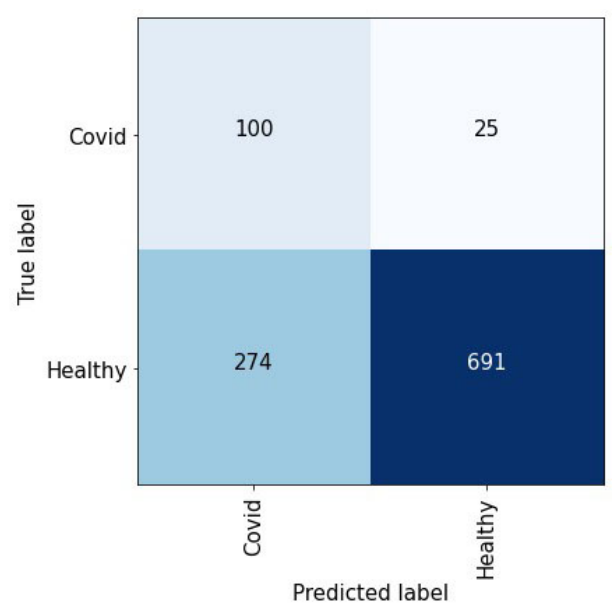

Fig. 7. The average validation confusion matrix consolidated from the five folds at sensitivity of $80 \%$. The specificity on the validation data was found to be $77.88 \%$.

sensitivity of $80 \%$ which implies that $80 \%$ of the positive class is correctly detected. We obtain the validation scores from each fold and report the overall performance. We can observe that at such a high sensitivity, the number of false negatives is significantly less than the total number of true negatives which is indicative of the detection performance.

\subsection{Comparison with methods from the Interspeech DiCOVA 2021 challenge}

We also entered our modified VGG-13 classifier into the "Diagnosing COVID-19 Using Acoustics" (DiCOVA) 2021 challenge [46], a special session of Interspeech 2021. Participants were tasked with a COVID19 audio classification task involving a blind test set of 233 audio samples. Our team placed eleventh out of 85 teams in the Track-1 challenge. Here, we describe methodologies and results for a number of the participants. All AUROC values given are for performance on the 5-fold cross validation. Team PANACEA [47] developed a method using Teager energy operator cepstral coefficients (TECCs) and a light gradient boosting machine (LightGBM) to achieve an AUROC of $69.80 \%$. In [48], a method is developed using support vector machines (SVMs) and long short-term memory (LSTM) networks in order to achieve and AUROC of 94.31\%. In [49], Random Forests, and SVMs are used in conjunction with the ComParE 2016 feature set. An AUROC of $73.29 \%$ was attained using this method. The study described in [50] demonstrated the use of logistic regression, random forests, and multilayer perceptrons to obtain an AUC value of $73.41 \%$. In [51], autore- 
gressive predictive coding (APC) is used in addition to an LSTM to achieve an AUC value of $76.45 \%$. The algorithm described in [52] only achieved an AUC of $47.28 \%$ but uses a unique encoder-decoder methodology for COVID-19 cough classification. In [53], the authors describe the use of a weighted SVM and the use of audio features such as super-vectors, formants, harmonics and MFCCs to attain an AUROC of 71.7\%. In [54], the authors use a Resnet 18 model for classification and evaluate performance of their algorithm between samples from male and female participants. This algorithm achieved an AUC of $61.90 \%$. The study described in [55] reported an AUROC of about 73.4\% using handcrafted features and an SVM classifier. In [56], the authors use an SVM classifier with high-dimensional acoustic features, as well as a CNN with log-Mel spectrograms. These models are fused in order to achieve an AUROC of $81 \%$. Our approach can perform COVID19 detection with an average AUROC of $82.23 \%$. We identify COVID-19 samples with an accuracy of $80 \%$ and a false positive rate of $17 \%$.

\section{Conclusions}

In this paper, we described system designs that was adopted for the DiCOVA 2021 dataset. We first explored the use of fully connected and pruned neural networks for COVID-19 cough detection. For the fully connected neural networks, we obtain an average validation accuracy of $65 \%$ on the 5 folds provided. In order to reduce complexity, we also explore the use of pruned neural networks. Using simulations, we demonstrate that the test accuracy of a network pruned by $50 \%$ (a significant reduction of weights) reduces only by $5 \%$. The pruned network is represented by half the number of parameters compared to the fully connected neural network. We then explored the use of convolutional architectures namely the VGG-13 for detection. In particular, we utilized a modified VGG-13 neural network with combined cross entropy and focal loss, operating on the log-mel spectrogram to learn to discriminate between the spectral signatures of coughs from a finite set of COVID-19 positive and healthy patients. We improved the overall generalization performance by: (i) performing data-augmentation using the COVID-19 positive cough sounds from the crowd sourced COUGHVID dataset; and (ii) using ensembles of two VGG-13 models, wherein the former was trained using the cross-entropy loss and the latter was trained using the focal loss. With these key modifications to the predictive modeling pipeline, our approach achieved high validation AUROCs (82.23\%) and blind test AUROCs $(78.3 \%)$, thereby encouraging the potential use of such models to support rapid diagnosis in clinical settings. With additional tuning of features and integrated loss functions, we anticipate further improvements on our results.

\section{Acknowledgments}

This work was funded by the REU supplement NSF NCSS/SenSIP I/UCRC Award number 1540040. We would also like to thank the authors in [57] for their insightful suggestions on model development.

\section{References}

[1] "Johns hopkins university, coronavirus resource center," https: //coronavirus.jhu.edu., accessed: 2021-01-21.

[2] Imran A, Posokhova I, Qureshi HN, Masood U, Riaz MS, Ali K, John CN, Hussain MI, Nabeel M. AI4COVID-19: AI enabled preliminary diagnosis for COVID-19 from cough samples via an app. Informatics in Medicine Unlocked. 2020 Jan 1; 20: 100378

[3] Sharma N, Krishnan P, Kumar R, Ramoji S, Chetupalli SR Ghosh PK, Ganapathy S. Coswara - A Database of Breathing, Cough, and Voice Sounds for COVID-19 Diagnosis. arXiv preprint arXiv:2005.10548. 2020 May 21.

[4] Orlandic L, Teijeiro T, Atienza D. The COUGHVID crowdsourcing dataset, a corpus for the study of large-scale cough analysis algorithms. Scientific Data. 2021 Jun 23; 8(1): 1-0.

[5] Shanthamallu US, Spanias A, Tepedelenlioglu C, Stanley M. A brief survey of machine learning methods and their sensor and IoT applications. In 2017 8th International Conference on Information, Intelligence, Systems \& Applications (IISA). IEEE. 2017 Aug 27, pp. 1-8.

[6] Spanias AS. Speech coding: A tutorial review. Proceedings of the IEEE. 1994 Oct; 82(10): 1541-82.

[7] Wichern G, Xue J, Thornburg H, Mechtley B, Spanias A. Segmentation, indexing, and retrieval for environmental and natural sounds. IEEE Transactions on Audio, Speech, and Language Processing. 2010 Feb 8; 18(3): 688-707.

[8] Loizou PC, Spanias AS. High-performance alphabet recognition. IEEE Transactions on Speech and Audio Processing. 1996 Nov; 4(6): 430-45.

[9] Shanthamallu US, Rao S, Dixit A, Narayanaswamy VS, Fan J, Spanias A. Introducing machine learning in undergraduate DSP classes. InICASSP 2019-2019 IEEE International Conference on Acoustics, Speech and Signal Processing (ICASSP). IEEE. 2019 May 12, pp. 7655-7659.

[10] Yang CH, Qi J, Chen SY, Chen PY, Siniscalchi SM, Ma X, Lee $\mathrm{CH}$. Decentralizing feature extraction with quantum convolutional neural network for automatic speech recognition. In ICASSP 2021-2021 IEEE International Conference on Acoustics, Speech and Signal Processing (ICASSP). IEEE. 2021 Jun 6, pp. 6523-6527.

[11] Ahmadi S, Spanias AS. Cepstrum-based pitch detection using a new statistical V/UV classification algorithm. IEEE Trans- 
actions on Speech and Audio Processing. 1999 May; 7(3): 333-8.

[12] Uehara G, Spanias A, Clark W. Quantum information processing algorithms with emphasis on machine learning. International Conference on Information, Intelligence, Systems \& Applications (IISA). IEEE. July 2021

[13] Coppock H, Gaskell A, Tzirakis P, Baird A, Jones L, Schuller B. End-to-end convolutional neural network enables COVID19 detection from breath and cough audio: A pilot study. BMJ Innovations. 2021 Apr 1; 7(2).

[14] Deshpande G, Schuller B. An overview on audio, signal, speech, \& language processing for covid-19. arXiv preprint arXiv:2005.08579. 2020 May 18.

[15] Chu HY, Englund JA, Starita LM, Famulare M, Brandstetter E, Nickerson DA, Rieder MJ, Adler A, Lacombe K, Kim AE, Graham C. Early detection of Covid-19 through a citywide pandemic surveillance platform. New England Journal of Medicine. 2020 Jul 9; 383(2): 185-7.

[16] Qian K, Schmitt M, Zheng H, Koike T, Han J, Liu J, Ji W, Duan J, Song M, Yang Z, Ren Z. Computer Audition for Fighting the SARS-CoV-2 Corona Crisis-Introducing the Multi-task Speech Corpus for COVID-19. IEEE Internet of Things Journal. 2021 Mar 22.

[17] Deshmukh S, Al Ismail M, Singh R. Interpreting glottal flow dynamics for detecting COVID-19 from voice. InICASSP 2021-2021 IEEE International Conference on Acoustics, Speech and Signal Processing (ICASSP). IEEE. 2021 Jun 6 , pp. 1055-1059.

[18] Han J, Brown C, Chauhan J, Grammenos A, Hasthanasombat A, Spathis D, Xia T, Cicuta P, Mascolo C. Exploring Automatic COVID-19 Diagnosis via voice and symptoms from Crowdsourced Data. InICASSP 2021-2021 IEEE International Conference on Acoustics, Speech and Signal Processing (ICASSP). IEEE. 2021 Jun 6, pp. 8328-8332.

[19] Rodriguez CR, Angeles D, Chafloque R, Kaseng F, Pandey B Deep Learning Audio Spectrograms Processing to the Early COVID-19 Detection. In 2020 12th International Conference on Computational Intelligence and Communication Networks (CICN). IEEE. 2020 Sep 25, pp. 429-434.

[20] Berisha V, Wisler A, Hero AO, Spanias A. Empirically estimable classification bounds based on a nonparametric divergence measure. IEEE Transactions on Signal Processing. 2015 Sep 10; 64(3): 580-91.

[21] Kushwaha S, Bahl S, Bagha AK, Kulwinder Singh P, Javaid M, Haleem A, Singh RP. Significant applications of machine learning for COVID-19 pandemic. Journal of Industrial Integration and Management. 2020.

[22] Esposito M, Rao S, Narayanaswamy S, Spanias A. Covid-19 detection using audio spectral features and machine learning. Asilomar Conference on Circuits, Systems and Computers. IEEE. Oct 2021.

[23] Laguarta J, Hueto F, Subirana B. COVID-19 artificial intelligence diagnosis using only cough recordings. IEEE Open Journal of Engineering in Medicine and Biology. 2020 Sep 29; 1: 275-81.

[24] Quatieri TF, Talkar T, Palmer JS. A framework for biomarkers of COVID-19 based on coordination of speech-production subsystems. IEEE Open Journal of Engineering in Medicine and Biology. 2020 May 29; 1: 203-6.

[25] Rao S, Narayanswamy V, Esposito M, Thiagarajan J, Spanias A. Deep Learning with hyper-parameter tuning for COVID-19 Cough Detection, IEEE IISA, 2021 July.

[26] Rao S, Muniraju G, Tepedelenlioglu C, Srinivasan D, Tamizhmani G, Spanias A. Dropout and Pruned Neural Networks for
Fault Classification in Photovoltaic Arrays. IEEE Access. 2021 Aug 30 .

[27] Rao S, Katoch S, Narayanaswamy V, Muniraju G, Tepedelenlioglu C, Spanias A, Turaga P, Ayyanar R, Srinivasan D. Machine learning for solar array monitoring, optimization, and control. Synthesis Lectures on Power Electronics. 2020 Aug 31; 7(1): 1-91.

[28] Frankle J, Carbin M. The lottery ticket hypothesis: Finding sparse, trainable neural networks. ICLR., 2019 May.

[29] Narayanaswamy VS, Katoch S, Thiagarajan JJ, Song H, Spanias A. Audio Source Separation via Multi-Scale Learning with Dilated Dense U-Nets. arXiv preprint arXiv:1904.04161. 2019 Apr 8

[30] Simonyan K, Zisserman A. Very deep convolutional networks for large-scale image recognition. arXiv preprint arXiv: 1409. 1556. 2014 Sep 4.

[31] Huzaifah M. Comparison of time-frequency representations for environmental sound classification using convolutional neura networks. arXiv preprint arXiv:1706.07156. 2017 Jun 22.

[32] Han R, Huang L, Jiang H, Dong J, Peng H, Zhang D. Early clinical and CT manifestations of coronavirus disease 2019 (COVID-19) pneumonia. American Journal of Roentgenology. 2020 Aug; 215(2): 338-43.

[33] WHO, "Clinical management of severe acute respiratory infection (sari) when covid-19 disease is suspected: interim guidance, 13 march 2020," World Health Organization, Tech. Rep., 2020.

[34] Maghded HS, Ghafoor KZ, Sadiq AS, Curran K, Rawat DB Rabie K. A novel AI-enabled framework to diagnose coronavirus COVID-19 using smartphone embedded sensors: design study. In 2020 IEEE 21st International Conference on Information Reuse and Integration for Data Science (IRI). IEEE. 2020 Aug 11, pp. 180-187.

[35] Han J, Qian K, Song M, Yang Z, Ren Z, Liu S, Liu J, Zheng H, Ji W, Koike T, Li X. An early study on intelligent analysis of speech under COVID-19: Severity, sleep quality, fatigue, and anxiety. arXiv preprint arXiv:2005.00096. 2020 Apr 30.

[36] Faezipour M, Abuzneid A. Smartphone-based self-testing of COVID-19 using breathing sounds. Telemedicine and eHealth. 2020 Oct 1; 26(10): 1202-5.

[37] Davis S, Mermelstein P. Comparison of parametric representations for monosyllabic word recognition in continuously spoken sentences. IEEE Transactions on Acoustics, Speech, and Signal Processing. 1980 Aug; 28(4): 357-66.

[38] Narayanaswamy VS, Thiagarajan JJ, Song H, Spanias A. Designing an effective metric learning pipeline for speaker diarization. InICASSP 2019-2019 IEEE International Conference on Acoustics, Speech and Signal Processing (ICASSP). IEEE. 2019 May 12, pp. 5806-5810.

[39] Laguarta J, Hueto F, Rajasekaran P, Sarma S, Subirana B. Longitudinal speech biomarkers for automated alzheimer's detection.

[40] He K, Zhang X, Ren S, Sun J. Deep residual learning for image recognition. In Proceedings of the IEEE Conference on Computer Vision and Pattern Recognition. 2016, pp. 770-778.

[41] Chaudhari G, Jiang X, Fakhry A, Han A, Xiao J, Shen S, Khanzada A. Virufy: Global applicability of crowdsourced and clinical datasets for AI detection of COVID-19 from cough. arXiv preprint arXiv:2011.13320. 2020 Nov 26.

[42] Spanias A, Painter T, Atti V. Audio signal processing and coding. John Wiley \& Sons; 2006 Sep 11.

[43] Ioffe S, Szegedy C. Batch normalization: Accelerating deep network training by reducing internal covariate shift. InInternational Conference on Machine Learning. PMLR. 2015 Jun 
1, pp. 448-456.

[44] Lin TY, Goyal P, Girshick R, He K, Dollár P. Focal loss for dense object detection. In Proceedings of the IEEE International Conference on Computer Vision. 2017, pp. 2980-2988.

[45] Zhang H, Cisse M, Dauphin YN, Lopez-Paz D. mixup: Beyond empirical risk minimization. arXiv preprint arXiv:1710.09412. 2017 Oct 25

[46] Muguli A, Pinto LRN, Sharma N, Krishnan P, Ghosh PK, Kumar R, Bhat S, Chetupalli SR, Ganapathy S, Ramoji S, Nanda V. DiCOVA Challenge: Dataset, Task, and Baseline System for COVID-19 Diagnosis Using Acoustics. Proc. Interspeech 2021.

[47] Kamble MR, Gonzalez-Lopez JA, Grau T, Espin JM, Cascioli L, Huang Y, Gomez-Alanis A, Patino J, Font R, Peinado AM, Gomez AM. PANACEA cough sound-based diagnosis of COVID-19 for the DiCOVA 2021 Challenge. Interspeech 2021.

[48] Karas V, Schuller BW. Recognising Covid-19 from Coughing Using Ensembles of SVMs and LSTMs with Handcrafted and Deep Audio Features. Proc. Interspeech 2021. 2021: 911-5.

[49] Södergren I, Nodeh MP, Chhipa PC, Nikolaidou GK, Kovács G. Detecting COVID-19 from audio recording of coughs using Random Forests and Support Vector Machines. Proc. Interspeech 2021. 2021: 916-20.

[50] Das RK, Madhavi M, Li H. Diagnosis of COVID-19 using Auditory Acoustic Cues. Proc. Interspeech 2021. 2021: 921-5.

[51] Harvill J, Wani YR, Hasegawa-Johnson M, Ahuja N, Beiser D, Chestek D. Classification of COVID-19 from Cough Using Autoregressive Predictive Coding Pretraining and Spectral Data Augmentation. Proc. Interspeech 2021. 2021: 926-30.

[52] Deshpande G, Schuller BW. The DiCOVA 2021 Challenge An Encoder-Decoder Approach for COVID-19 Recognition from Coughing Audio. Proc. Interspeech 2021. 2021: 931-5.
[53] Ritwik KV, Kalluri SB, Vijayasenan D. COVID-19 Detection from Spectral features on the DiCOVA Dataset. Proc. Interspeech 2021. 2021: 936-40.

[54] Mallol-Ragolta A, Cuesta H, Gómez E, Schuller BW. CoughBased COVID-19 Detection with Contextual Attention Convolutional Neural Networks and Gender Information. Proc. Interspeech 2021. 2021: 941-5.

[55] Bhosale S, Tiwari U, Chakraborty R, Kopparapu SK. Contrastive Learning of Cough Descriptors for Automatic COVID19 Preliminary Diagnosis. Proc. Interspeech 2021. 2021: 94650.

[56] Avila F, Poorjam AH, Mittal D, Dognin C, Muguli A, Kumar R, Chetupalli SR, Ganapathy S, Singh M. Investigating Feature Selection and Explainability for COVID-19 Diagnostics from Cough Sounds. Proc. Interspeech 2021. 2021: 951-5.

[57] Iqbal T, Kong Q, Plumbley MD, Wang W. General-purpose audio tagging from noisy labels using convolutional neural networks. In Proceedings of the Detection and Classification of Acoustic Scenes and Events 2018 Workshop (DCASE2018). 2018, pp. 212-216. Tampere University of Technology.

[58] Chen T, Guestrin C. Xgboost: A scalable tree boosting system. In Proceedings of the 22nd Acm Sigkdd International Conference on Knowledge Discovery and Data Mining. 2016 Aug 13, pp. $785-794$

[59] Simonyan K, Zisserman A. Very deep convolutional networks for large-scale image recognition. arXiv preprint arXiv:1409. 1556. 2014 Sep 4.

[60] Iqbal T, Kong Q, Plumbley M, Wang W. Stacked convolutional neural networks for general-purpose audio tagging. DCASE2018 Challenge. 2018 Sep. 